20

\title{
Микрокристаллические алмазные порошки как перспективные объекты для генерации многочастотного вынужденного комбинационного рассеяния*
}

\author{
(C) B.C. Горелик ${ }^{1,2, \uparrow}$, А.В. Скрабатун ${ }^{1,2}$, Dongxue $B i^{1,2}$ \\ ${ }^{1}$ Физический институт им. П.Н. Лебедева РАН, \\ 119991 Москва, Россия \\ ${ }^{2}$ Московский государственный технический университет им. Н.Э. Баумана, \\ 105005 Москва, Россия \\ I e-mail: gorelik@sci.lebedev.ru
}

Поступила в редакцию 18.11.2018 г.

В окончательной редакции 11.01.2019 г.

Принята к публикации 31.01.2019 г.

\begin{abstract}
Исследованы закономерности комбинационного рассеяния света в микрокристаллических алмазных порошках в зависимости от размеров алмазных микрорезонаторов в диапазоне 1-600 $\mu$ m. Наблюдаемый эффект аномально высокой интенсивности спонтанного комбинационного рассеяния в алмазных микрорезонаторах объясняется „пленением“ в них электромагнитного излучения, длина волны которого меньше размеров микрокристаллов алмаза. Вследствие „пленения“ фотонов в алмазных микрорезонаторах повышается плотность электромагнитной энергии для возбуждающего и вторичного излучений. Высокая добротность фундаментальной оптической моды в колебательном спектре алмаза и аномальное возрастание интенсивности комбинационного рассеяния в алмазных микрорезонаторах открывают возможности для наблюдения низкопорогового вынужденного многочастотного комбинационного рассеяния в микрокристаллических алмазных порошках. Использование в качестве возбуждающего излучения линий генераций импульсного твердотельного лазера $\mathrm{YAG}: \mathrm{Nd}^{3+}(\lambda=1064 \mathrm{~nm})$ и его оптических гармоник $(\lambda=1064$, $532,355,266 \mathrm{~nm}$ ) открывает возможность для создания линейки эквидистантных (по сдвигу частоты) генераторов лазерного излучения от ультрафиолетовой области до терагерцового диапазона, перспективного для исследования биологических и медицинских объектов.
\end{abstract}

DOI: $10.21883 /$ OS.2019.05.47661.10-19

\section{Введение}

Кристаллы алмаза являются уникальными объектами для исследований методом комбинационного рассеяния (КР) света вследствие рекордно высоких показателей механических и оптических свойств. Природные алмазы стали изучаться методом КР практически сразу после открытия этого явления [1]. Регистрация спектров КР синтетических алмазов позволила оптимизировать технологию их синтеза и обеспечила возможность получения совершенных и достаточно крупных образцов $[2,3]$.

Алмаз, являющийся основной высокобарической фазой углерода, кристаллизуется в кубической сингонии и имеет пространственную группу $O_{h}^{7}$ симметрии с двумя атомами углерода в примитивной ячейке [3]. Согласно результатам теоретико-группового анализа $[4,5]$, в спектре длинноволновых оптических фононов кристалла алмаза присутствует лишь одна трехкратно вырожденная мода $\left(v=1332 \mathrm{~cm}^{-1}\right)$ типа $F_{2 g}$, активная в спектре КР,

\footnotetext{
* The 22nd Annual Conference Saratov Fall Meeting 2018 (SFM'18): VI International Symposium „Optics and Biophotonics“ and XXII International School for Junior Scientists and Students on Optics, Laser Physics \& Biophotonics, September 24-29, 2018, Saratov, Russia. https://www.sgu.ru/structure/fiz/saratov-fall-meeting/previousconferences/sara
}

но запрещенная для процессов однофотонного поглощения в ИК области. Методом КР сравнительно легко можно идентифицировать присутствие алмазов в ювелирных изделиях и в гетерогенных фазах. Теория [6,7] предсказывает, что уменьшение размеров кристаллов алмаза приводит к коротковолновому сдвигу частоты фундаментальной $F_{2 g}$-моды и к деформации соответствующей спектральной формы сигнала спонтанного КР. В настоящее время весьма актуальной является задача установления закономерностей спектров КР в нано- и микрокристаллических частицах алмазов [7-11], а также в алмазных пленках, получаемых методом химического осаждения из газовой фазы (chemical vapor deposition CVD) $[12,13]$. При возбуждении КР в алмазных микрои нанокристаллах различными источниками лазерного излучения наблюдаются дополнительные полосы, которые могут принадлежать примесям графита [14] и аморфного углерода [15]. Увеличение длины волны возбуждающего излучения приводит к появлению в спектре KP CVD-пленок микро- и нанокристаллов алмаза многочисленных „биений“ и высокоинтенсивного фона в области частот до фундаментальной моды $1332 \mathrm{~cm}^{-1}[13,16,17]$.

Кристаллы алмаза характеризуются сравнительно высоким коэффициентом комбинационного уси- 

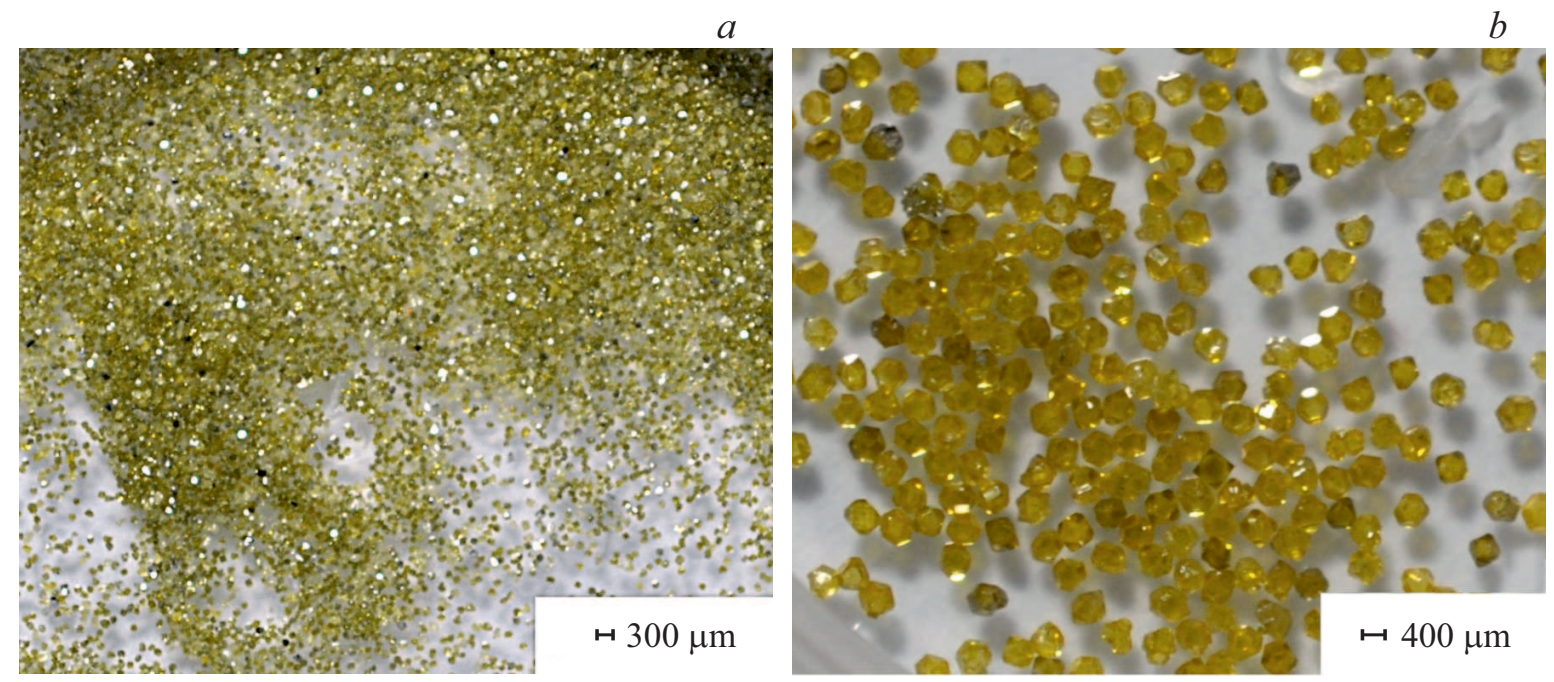

Рис. 1. Фотографии искусственных микрокристаллов алмаза; $a-150-180 \mu \mathrm{m} ; b-425-600 \mu \mathrm{m}$.

ления при вынужденном комбинационном рассеянии света (ВКР): $\beta \sim 10 \mathrm{~cm} / \mathrm{GW}$ при накачке лазером $\mathrm{YAG}: \mathrm{Nd}^{3+}(\lambda=1064 \mathrm{~nm})[18]$. Они характеризуются также рекордным коэффициентом теплопроводности $(\sim 2000 \mathrm{~W} / \mathrm{m} \cdot \mathrm{K})[19]$, большим частотным сдвигом фундаментальной моды в спектре ВКР $\left(v=1332 \mathrm{~cm}^{-1}\right)$. Все эти особенности, а также широкий диапазон прозрачности кристаллов алмаза (от дальнего ультрафиолета до радиодиапазона) [20] делают этот кристалл весьма перспективным для генерации многочастотного ВКР и наблюдения параметрического четырехволнового рассеяния света.

Исследования ВКР в искусственных алмазах активно проводятся в последние годы рядом научных коллективов [18-23]. При этом до последнего времени возбуждение ВКР осуществлялось на искусственных алмазных пленках и тонких пластинках искусственных алмазов. Разрабатываются лазеры на основе синтетических CVD-пленок $[24,25]$, позволяющие достигать выходной мощности $24.5 \mathrm{~W}$ [21].

Целью настоящей работы является исследование закономерностей спектров КР света в поликристаллических алмазных порошках в зависимости от размеров микрочастиц алмазов. Решение такой задачи открывает возможность для генерации линейки частот лазерного излучения в широком спектральном диапазоне при ВКР в поликристаллических алмазных порошках.

\section{Экспериментальная часть}

В используемых кристаллических алмазных порошках (рис. $1, a, b)$ присутствовали микрочастицы близких размеров в виде многогранников - микрорезаноторов. Размер микрочастиц изменялся в диапазоне $d=1-600 \mu \mathrm{m}$.

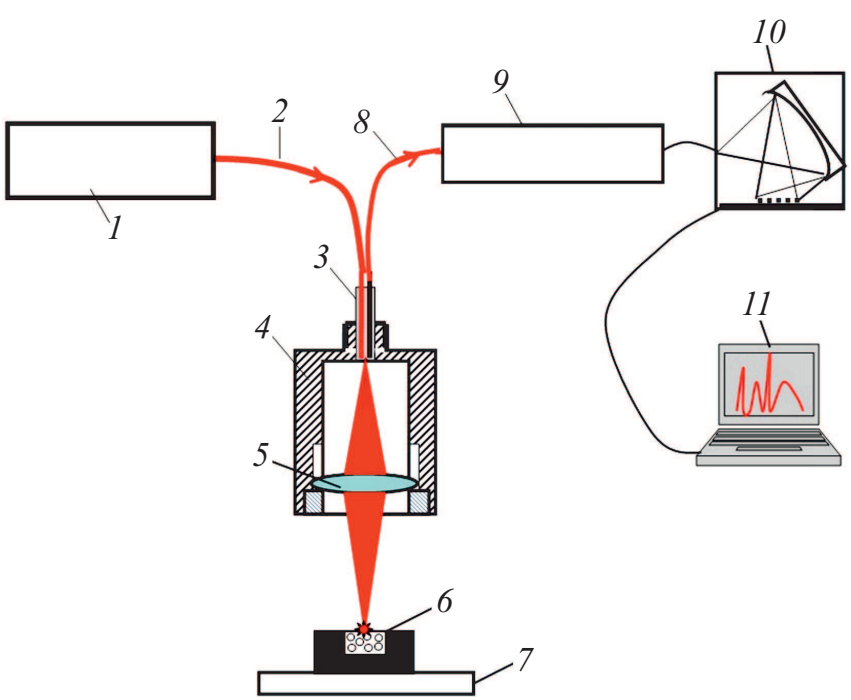

Рис. 2. Схема экспериментальной установки для наблюдения спонтанного КР в алмазных порошках: $1-$ зонд; $4-$ корпус; 5 - линза; 6 - кювета; 7 - подложка; 9 - фокусатор; $10-$ мини-спектрометр; 11 - компьютер.

В настоящей работе использовались кристаллические порошки с заданными размерами частиц: $1-2,10-20$, $150-180$ и $425-600 \mu \mathrm{m}$.

Для возбуждения и регистрации спектров спонтанного КР света была использована экспериментальная установка, схема которой приведена на рис. 2. В качестве источника возбуждающего излучения применялся лазер 1 , работающий в непрерывном режиме с длиной волны $\lambda_{0}=785 \mathrm{~nm}$ и мощностью $100 \mathrm{~mW}$. Лазерное излучение вводилось в первый световод 2 и зонд 3. С помощью линзы 5 это излучение фокусировалось на кювете 6 , заполненной микрокристаллическим алмазным порошком. 

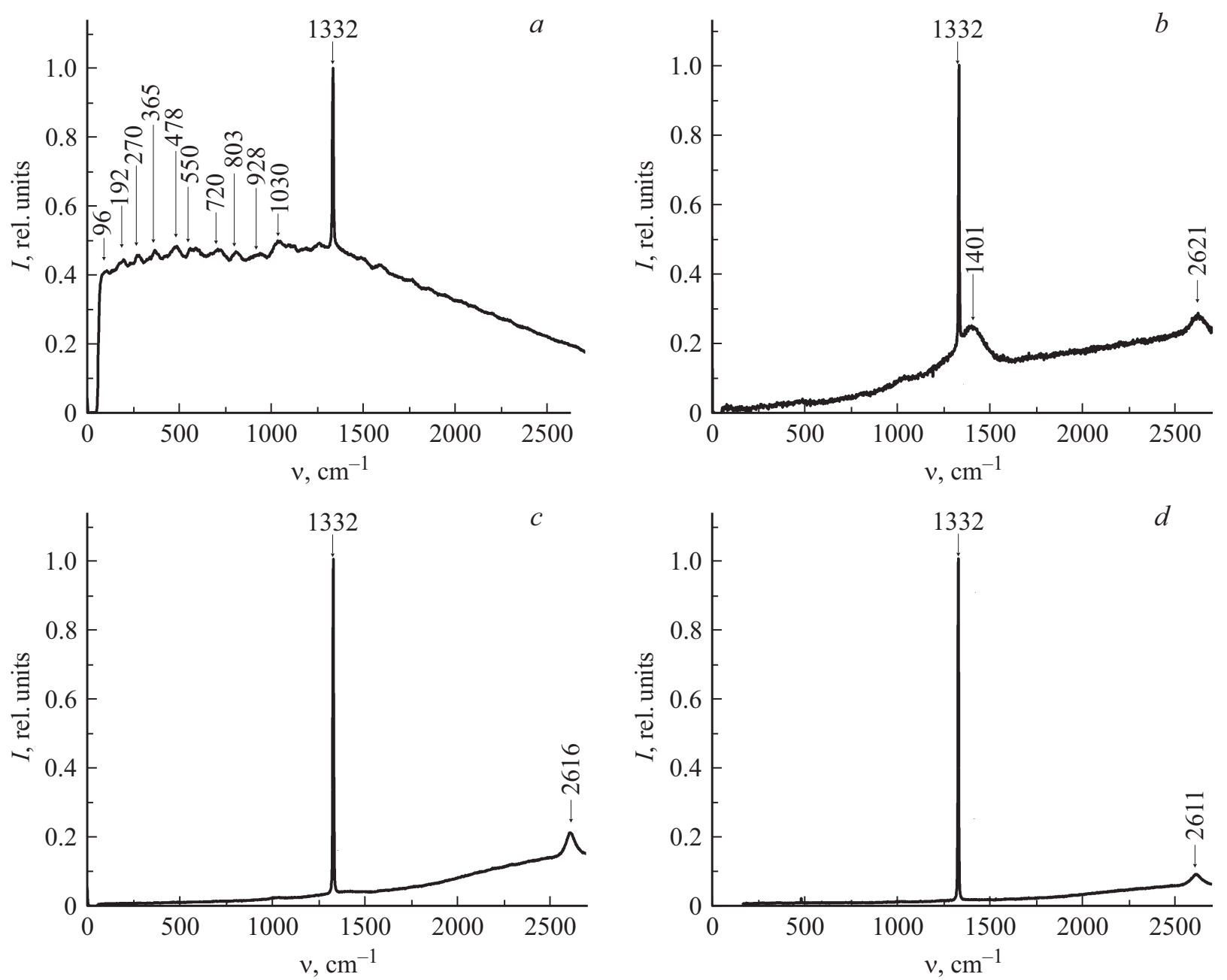

Рис. 3. Нормированные спектры КР микрокристаллических алмазных порошков с различными размерами зерен; $a-1-2 \mu \mathrm{m}$, $b-10-20 \mu \mathrm{m}, c-150-180 \mu \mathrm{m}, d-425-600 \mu \mathrm{m}$.

Возникающий в образце сигнал КР попадал на второй световод 8 , фильтровался в фокусаторе 9 с помощью „нотч-фильтра“ и поступал на вход мини-спектрометра типа BWS465-785H 10 с многоэлементным приемником. Компьютер 11 был предназначен для цифровой обработки и накопления спектров КР. Спектральное разрешение составляло $1 \mathrm{~cm}^{-1}$. Полный спектр КР микроалмазов в диапазоне $50-2750 \mathrm{~cm}^{-1}$ регистрировался при экспозициях $10-100 \mathrm{~s}$.

\section{Результаты экспериментальных исследований}

Зарегистрированные спектры КР в микрокристаллических алмазных порошках с различными размерами зерен представлены на рис. 3. Как видно из рис. 3, вид спектров КР в микрокристаллах алмазов существенно отличается от известных спектров КР первого порядка в крупных алмазах, состоящих только из одной линии с частотой $1332 \mathrm{~cm}^{-1}$. Для образца с размером зерен $d \sim 1 \mu \mathrm{m}$ в области низких частот наблюдаются многократные „биения“ и непрерывный фон по всей области спектра КР. В то же время в этом спектре (рис. 3,a) присутствует характерный пик с частотой $1332 \mathrm{~cm}^{-1}$, интенсивность которого сопоставима с интенсивностью непрерывного фона. При возрастании размера $d$ алмазных микрорезонаторов до $10 \mu \mathrm{m}$ интенсивность непрерывного фона уменьшалась, а фундаментального пика увеличивалась (рис. $3, b$ ). При этом биения интенсивности КР на частотах 96, 192, 270, 365,478, $550,720,803,928,1030 \mathrm{~cm}^{-1}$ не обнаруживались, но наблюдалось появление двух дополнительных широких полос с частотами максимумов $1401 \mathrm{~cm}^{-1}$ (рис. 3, $b$ ) и в области $2620 \mathrm{~cm}^{-1}$ (рис. $3, b-d$ ). При дальнейшем увеличении размера микрорезонаторов $(d=150-600 \mu \mathrm{m})$ непрерывный фон в спектре КР практически отсутствовал. При этом, кроме резкого интенсивного фундаментального пика $1332 \mathrm{~cm}^{-1}$, в обертонной области спектра наблюдался достаточно интенсивный пик в области с частотой $2620 \mathrm{~cm}^{-1}$. 
Длины волн и частоты комбинационных спутников, рассчитанные при эквидистантном частотном сдвиге на $1332 \mathrm{~cm}^{-1}$ от линий генерации $\lambda=1.064,0.532,0.355,0.266 \mu \mathrm{m}$. В скобках указаны номера стоксовых (S) и антистоксовых (AS) компонент

\begin{tabular}{|c|c|c|c|c|c|}
\hline \multicolumn{3}{|c|}{$\lambda_{01}=1.064 \mu \mathrm{m}\left(9398 \mathrm{~cm}^{-1}\right)$} & \multicolumn{3}{|c|}{$\lambda_{02}=0.532 \mu \mathrm{m}\left(18797 \mathrm{~cm}^{-1}\right)$} \\
\hline $\begin{array}{c}\text { Длина волны } \\
\lambda, \mu \mathrm{m}\end{array}$ & $\begin{array}{c}\text { Частота } v, \\
\mathrm{~cm}^{-1}\end{array}$ & $\begin{array}{c}\mathrm{S} \text { и } \mathrm{AS} \\
\text { спутники }\end{array}$ & $\begin{array}{c}\text { Длина волны } \\
\lambda, \mu \mathrm{m}\end{array}$ & $\begin{array}{c}\text { Частота } v, \\
\mathrm{~cm}^{-1}\end{array}$ & $\begin{array}{c}\mathrm{S} \text { и AS } \\
\text { спутники }\end{array}$ \\
\hline $\begin{array}{r}0.931 \\
0.829 \\
0.679 \\
0.623 \\
0.746 \\
0.575 \\
0.534 \\
1.240 \\
1.485 \\
1.851 \\
2.457 \\
3.652 \\
7.112 \\
135.135\end{array}$ & $\begin{array}{r}10738 \\
13394 \\
14726 \\
16058 \\
17390 \\
12062 \\
18722 \\
8066 \\
6734 \\
5402 \\
4070 \\
2738 \\
1406 \\
74\end{array}$ & $\begin{array}{c}\operatorname{AS}(1) \\
\operatorname{AS}(2) \\
\operatorname{AS}(3) \\
\operatorname{AS}(4) \\
\operatorname{AS}(5) \\
\operatorname{AS}(6) \\
\mathrm{AS}(7) \\
\mathrm{S}(1) \\
\mathrm{S}(2) \\
\mathrm{S}(3) \\
\mathrm{S}(4) \\
\mathrm{S}(5) \\
\mathrm{S}(6) \\
\mathrm{S}(7)\end{array}$ & $\begin{array}{l}0.497 \\
0.466 \\
0.439 \\
0.414 \\
0.393 \\
0.373 \\
0.355 \\
0.572 \\
0.620 \\
0.675 \\
0.742 \\
0.824 \\
0.925 \\
1.055\end{array}$ & $\begin{array}{r}20129 \\
21461 \\
22793 \\
24125 \\
25457 \\
26789 \\
28121 \\
17465 \\
16133 \\
14801 \\
13469 \\
12137 \\
10805 \\
9473\end{array}$ & $\begin{array}{l}\operatorname{AS}(1) \\
\operatorname{AS}(2) \\
\operatorname{AS}(3) \\
\operatorname{AS}(4) \\
\operatorname{AS}(5) \\
\operatorname{AS}(6) \\
\operatorname{AS}(7) \\
\mathrm{S}(1) \\
\mathrm{S}(2) \\
\mathrm{S}(3) \\
\mathrm{S}(4) \\
\mathrm{S}(5) \\
\mathrm{S}(6) \\
\mathrm{S}(7)\end{array}$ \\
\hline \multicolumn{3}{|c|}{$\lambda_{03}=0.355 \mu \mathrm{m}\left(28194 \mathrm{~cm}^{-1}\right)$} & \multicolumn{3}{|c|}{$\lambda_{04}=0.266 \mu \mathrm{m}\left(37594 \mathrm{~cm}^{-1}\right)$} \\
\hline $\begin{array}{c}\text { Длина волны } \\
\lambda, \mu \mathrm{m}\end{array}$ & $\begin{array}{c}\text { Частота } v, \\
\mathrm{~cm}^{-1}\end{array}$ & $\begin{array}{c}\mathrm{S} \text { и AS } \\
\text { спутники }\end{array}$ & $\begin{array}{c}\text { Длина волны } \\
\lambda, \mu \mathrm{m}\end{array}$ & $\begin{array}{c}\text { Частота } v, \\
\mathrm{~cm}^{-1}\end{array}$ & $\begin{array}{c}\mathrm{S} \text { и } \mathrm{AS} \\
\text { спутники }\end{array}$ \\
\hline $\begin{array}{l}0.338 \\
0.324 \\
0.310 \\
0.298 \\
0.287 \\
0.276 \\
0.266 \\
0.372 \\
0.392 \\
0.413 \\
0.437 \\
0.464 \\
0.495 \\
0.530\end{array}$ & $\begin{array}{l}29526 \\
30858 \\
32190 \\
33522 \\
34854 \\
36186 \\
37518 \\
26862 \\
25530 \\
24198 \\
22866 \\
21534 \\
20202 \\
18870\end{array}$ & $\begin{array}{c}\mathrm{AS}(1) \\
\mathrm{AS}(2) \\
\mathrm{AS}(3) \\
\mathrm{AS}(4) \\
\mathrm{AS}(5) \\
\mathrm{AS}(6) \\
\mathrm{AS}(7) \\
\mathrm{S}(1) \\
\mathrm{S}(2) \\
\mathrm{S}(3) \\
\mathrm{S}(4) \\
\mathrm{S}(5) \\
\mathrm{S}(6) \\
\mathrm{S}(7)\end{array}$ & $\begin{array}{l}0.257 \\
0.248 \\
0.240 \\
0.233 \\
0.226 \\
0.219 \\
0.213 \\
0.275 \\
0.286 \\
0.297 \\
0.310 \\
0.323 \\
0.338 \\
0.353\end{array}$ & $\begin{array}{l}38926 \\
40258 \\
41590 \\
42922 \\
44254 \\
45586 \\
46918 \\
36262 \\
34930 \\
33598 \\
32266 \\
30934 \\
29602 \\
28270\end{array}$ & $\begin{array}{c}\operatorname{AS}(1) \\
\operatorname{AS}(2) \\
\operatorname{AS}(3) \\
\operatorname{AS}(4) \\
\operatorname{AS}(5) \\
\operatorname{AS}(6) \\
\mathrm{AS}(7) \\
\mathrm{S}(1) \\
\mathrm{S}(2) \\
\mathrm{S}(3) \\
\mathrm{S}(4) \\
\mathrm{S}(5) \\
\mathrm{S}(6) \\
\mathrm{S}(7)\end{array}$ \\
\hline
\end{tabular}

\section{Обсуждение полученных результатов}

Согласно известной теории рассеяния Ми на сферических частицах [26], индикатриса рассеянного излучения света существенно видоизменяется в том случае, когда длина волны электромагнитного излучения, отнесенная к соответствующему показателю преломления, становится сравнимой или меньше размеров рассеивающих частиц. Алмазные микрочастицы характеризуются большим значением показателя преломления в видимом диапазоне длин волн $(n=2.4-2.5)$ [27]. Таким образом, исследуемые микрокристаллы алмазов удовлетворяют условиям для наблюдения резонансов Ми, а сами микрочастицы можно рассматривать как оптические резонаторы, обеспечивающие „пленение излучения в них мод типа шепчущей галереи. Соответственно наблюдаемое резкое возрастание эффек- тивности КР на фундаментальной моде $1332 \mathrm{~cm}^{-1}$ по сравнению с крупными образцами алмазов, исследуемых при 90-градусной геометрии рассеяния [3], можно объяснить „пленением“ электромагнитного излучения в алмазных микрочастицах и соответствующего резкого увеличения его плотности энергии. Наблюдаемый непрерывный фон и присутствие биений в низкочастотной области в спектре алмазов микронного размера (рис. 3,a) можно объяснить проявлением стоячих волн в микрорезонаторах, соответствующих акустическим и оптическим дисперсионным ветвям кристаллов алмаза. Пик с частотой $1401 \mathrm{~cm}^{-1}$, наблюдаемый в спектре КР (рис. 3,b) для микрочастиц с размером $d=10-20 \mu \mathrm{m}$, естественно отнести на счет двухфононного возбуждения, соответствующего критической точке акустической ветви на границе зоны Бриллюэна. 
Особый интерес представляет пик в области $2620 \mathrm{~cm}^{-1}$ (рис. $3, b-d$ ), расположенный вблизи обертона моды с частотой $1332 \mathrm{~cm}^{-1}$. Известно $[28,29]$, что в крупных монокристаллах алмаза наблюдался слабый резкий пик КР на частоте $2668 \mathrm{~cm}^{-1}$, интерпретируемый как связанное состояние двух фононов - бифонона с очень малой энергией связи. Для подтверждения такой интерпретации проводилось сопоставление наблюдаемого спектра КР второго порядка в алмазе с плотностью двухфононных состояний [27-29]. Соответственно пик в области $2620 \mathrm{~cm}^{-1}$ (рис. $3, b-d$ ) естественно объяснить как проявление связанного состояния двух оптических фононов (бифонона) с возросшей энергией связи $\left(\Delta=44 \mathrm{~cm}^{-1}\right)$ в условиях конфайнмента в алмазном микрорезонаторе. При этом интенсивность КР на бифононе только в пять раз ниже интенсивности КР на основном тоне.

Кристаллы алмаза прозрачны в широком спектральном диапазоне: от дальнего ультрафиолетового до радиоволнового излучения. Такие окна прозрачности обсуждаемого материала, а также сравнительно низкий порог ВКР делают алмаз одним из удобных объектов для наблюдения многочастотного ВКР. Выполненные к настоящему времени работы по наблюдению многочастотного ВКР в алмазных CVD-пленках имеют ограниченные возможности из-за небольшой толщины пленок, в результате чего возникает необходимость использовать высокую плотность мощности возбуждающего лазерного излучения, приводящую к светоиндуцированным процессам разрушения дорогостоящих образцов. С этой точки зрения микрокристаллические алмазные порошки имеют существенные преимущества, обусловленные большой эффективностью спонтанного КР и соответственно низким порогом ВКР-генерации, а также возможностью использования большого количества образцов различных размеров. Как показали наши эксперименты, наиболее перспективными микрокристаллическими алмазными порошками для возбуждения ВКР являются образцы с размерами $150-180 \mu \mathrm{m}$, характеризующиеся присутствием высокодобротной фундаментальной моды $1332 \mathrm{~cm}^{-1}$ в спектре КР.

В таблице приведены рассчитанные значения длин волн и частот линий генерации многочастотного ВКР в алмазах, возбуждаемого линией генерации $1.064 \mu \mathrm{m}$ твердотельного лазера $\mathrm{YAG}: \mathrm{Nd}^{3+}$ и его оптических гармоник с длинами волн 0.532, 0.355 и $0.266 \mu \mathrm{m}$. Как показывают результаты исследований многочастотного ВКР в конденсированных средах, наиболее эффективное возбуждение ВКР осуществляется при использовании ультракоротких лазерных импульсов с длительностью 20-80 ps. Согласно данным таблицы, в спектре многочастотного ВКР ожидается присутствие семи стоксовых (S) и антистоксовых (AS) компонент, эквидистантно сдвинутых друг относительно друга на частоту фундаментальной моды $\Delta v=1332 \mathrm{~cm}^{-1}$. Отметим, что при возбуждении ВКР в алмазах импульсным ИК излучением $(\lambda=1.064 \mu \mathrm{m})$ ожидает- ся генерация в среднем и дальнем ИК диапазонах, вплоть до длины волны $135 \mu \mathrm{m}\left(v=74 \mathrm{~cm}^{-1}\right)$, соответствующей терагерцовой области спектра, перспективной для исследований биологических и медицинских объектов.

\section{Заключение}

Таким образом, в настоящей работе зарегистрированы спектры КР микрокристаллических алмазных порошков с размерами частиц $d=1-600 \mu \mathrm{m}$. Микрокристаллы алмазов, присутствующие в порошке, имели вид микрорезонаторов, обеспечивающих пленение возбуждающего излучения и КР. В результате полный спектр спонтанного КР в кристаллическом алмазном порошке в диапазоне $50-2750 \mathrm{~cm}^{-1}$ удавалось регистрировать при небольших экспозициях: 10-100s. При размерах алмазных зерен $d \sim 1 \mu \mathrm{m}$ в спектре КР присутствовал большой непрерывный фон, который является результатом проявления однофононных и двухфононных акустических процессов. При переходе к образцам с размером $d=10-20 \mu \mathrm{m}$ в спектре КР, кроме фундаментальной моды с частотой $1332 \mathrm{~cm}^{-1}$, были обнаружены дополнительные полосы 1401 и $2620 \mathrm{~cm}^{-1}$, обусловленные двухфононными переходами и проявлением связанного состояния двух фононов с большой энергией связи. При этом интенсивность в спектре КР пика, соответствующего связанному состоянию $\left(2620 \mathrm{~cm}^{-1}\right)$, оказалась всего лишь в пять раз меньше интенсивности КР на фундаментальной моде. Для образцов микрокристаллов с размерами $d=150-600 \mu \mathrm{m}$ в спектре непрерывный фон практически отсутствовал, и интенсивность КР на фундаментальной моде существенно превышала аналогичную величину для микрокристаллов меньших размеров.

На основании проведенных исследований спонтанного КР в микрокристаллических алмазных порошках сделан вывод о перспективности их использования для возбуждения линейки частот при возбуждении многочастотного ВКР интенсивными лазерными импульсами наносекундного или пикосекундного диапазонов с различными длинами волн: $\lambda=1.064,0.532,0.355$ и $0.266 \mu \mathrm{m}$. При этом ожидается присутствие большого числа линий генерации в широкой области спектра (от дальней ИК области до ультрафиолетового диапазона), эквидистантно сдвинутых по частоте на $1332 \mathrm{~cm}^{-1}$.

\section{Финансирование работы}

Работа выполнена при поддержке РФФИ (гранты № 16-08-00618, 16-02-00488, 18-02-00181, 18-32-00259) и China Scholarship Council. 


\section{Список литературы}

[1] Krishnan R.S. // Proc. Indian Acad. Sci. Sec. A. 1947. V. 26. P. 399-418. doi 10.1007/BF03170898

[2] Bundy F.P., Hall H.T., Strong H.M., Wentorf R.H. // Nature. 1955. V. 176. P. 51-55. doi 10.1038/176051a0

[3] Knight D.S., White W.B. // J. Mater. Res. 1989. V. 4. N 2. P. 385-393. doi 10.1557/JMR.1989.0385

[4] Solin S.A., Ramdas A.K. // Phys. Rev. B. 1970. V. 1. N 4. P. 1687-1698. doi 10.1103/PhysRevB.1.1687

[5] Orwa J.O., Nugent K.W., Jamieson D.N., Prawer S. // Phys. Rev. B. 2000. V. 62. N 9. P. $5461-5472$. doi 10.1103/PhysRevB.62.5461

[6] Yoshikawa M., Mori Y., Maegawa M., Katagiri G., Ishida H., Ishitani A. // Appl. Phys. Lett. 1993. V. 62. N 24. P. 3114-3116. doi 10.1063/1.109154

[7] Аргунов К.П., Горелик В.С., Резник Б.И., Ротнер Ю.М., Файзуллов Т.Ф. // Краткие сообщения по физике ФИАН. 1991. № 9. C. 21-24; Argunov K.P., Gorelik V.S., Reznik B.I., Rotner Yu.M., Faizullov T.F. // Sov. Phys. Lebedev Inst. Rep. 1991. N 9. P. 18.

[8] Palnichenko A.V., Jonas A.M., Charlier J.-C., Aronin A.C., Issl J.-P. // Nature. 1999. V. 402. P. 162-165. doi 10.1038/46000

[9] Nasdala L., Steger S., Reissner C. // Lithos. 2016. V. 265. P. 317-327. doi 10.1016/j.lithos.2016.03.009

[10] Углов В.В., Шиманский В.И., Русальский Д.П., Самцов М.П. // ЖПС. 2008. Т. 75. № 4. С. 524-528; Uglov V.V., Shimanski V.I., Rusalsky D.P., Samtsov M.P. // J. Appl. Spectrosc. 2008. V. 75. N 4. P. 546-549.

doi 10.1007/s10812-008-9078-6

[11] Горелик В.С., Иго А.В., Миков С.Н. // ЖЭТФ. 1996. № 109. C. 2141-2149; Gorelik V.S., Igo A.V., Mikov S.N. // JETP. 1996. V. 82. N 6. P. 1154-1158.

[12] Lee K.C., Sussman B.J., Sprague M.R., Michelberger P., Reim K.F., Nunn J., Langford N.K., Bustard P.J., Jaksch D., Walmsley I.A. // Nature Photonics. 2011. V. 6. P. 11-14. doi 10.1038/NPHOTON.2011.296

[13] May P.W., Smith J.A., Rosser K.N. // Diamond Relat. Mater. 2008. V. 17. P. 199-203. doi 10.1557/PROC-1039-P15-02

[14] Okada K., Kanda H., Komatsu S., Matsumoto S. // J. Appl. Phys. 2000. V. 88. N 8. P. 1674-1678. doi 10.1063/1.373870

[15] Ferrari A.C., Robertson J. // Phys. Rev. B. 2001. V. 63. P. 121405. doi 10.1103/PhysRevB.63.121405

[16] Gyollai I., Gucsik A., Veres M., Koós M., Nagy S., Bérczi S. // Spectrosc. Lett. 2012. V. 45. N 2. P. 151-155. doi 10.1080/00387010.2011.627527

[17] May P.W., Ludlow W.J., Hannaway M., Heard P.J., Smith J.A., Rosser K.N. // Diamond Relat. Mater. 2008. V. 17. P. 105-117. doi 10.1016/j.diamond.2007.11.005

[18] Williams R.J., Kitzler O., Bai Z., Sarang S., Jasbeer H., McKay A., Antipov S., Sabella A., Lux O., Spence D.J., Mildren R.P. // J. Selected Topics in Quantum Electronics. 2018. V. 24. N 5. P. 1602214. doi 10.1109/JSTQE.2018.2827658

[19] Olson J.R., Pohl R.O., Vandersande J.W., Zoltan A., Anthony T.R., Banholzer W.F. // Phys. Rev. B. Condens. Matter. 1993. V. 47. N 22. P. $14850-14856$. doi 10.1103/PhysRevB.47.14850

[20] Kaminskii A.A., Ral'chenko V.G., Konov V.I. // Letter JETP. 2004. V. 80. N 4. P. 298-301. doi 10.1134/1.1813684
[21] Lux O., Ralchenko V.G., Bolshakov A.P., Konov V.I., Sharonov G.V., Shirakawa A., Yoneda H., Rhee H., Eichler H.J., Mildren R.P., Kaminskii A.A. // Laser Phys. Lett. 2014. V. 11. P. 086101. doi 10.1088/1612-2011/11/8/086101

[22] Granados E., Spence D.J., Mildren R.P. // Optics Express. 2011. V. 19. N 11. P. 10857-10863. doi 10.1364/OE.19.010857

[23] Kaminskii A.A., Hemley R.J., Lai J., Yan C.S., Mao H.K., Ralchenko V.G., Eichler H.J., Rhee H. // Laser Phys. Lett. 2007. V. 4. N 5. P. 350-353. doi 10.1002/lapl.200610127

[24] Mildren R.P., Butler J.E., Rabeau J.R. // Optics Express. 2008. V. 16. N 23. P. 18950-18955. doi 10.1364/OE.16.018950

[25] Feve J.-P.M., Shortoff K.E., Bohn M.J., Brasseur J.K. // Optics Express. 2011. V. 19. N 2. P. 913-922. doi 10.1364/OE.19.000913

[26] Bohren C.F., Huffman D.R. Absorption and Scattering of Light by Small Particles. Wiley-Interscience, 1983. 533 p.; Борен К., Хафмен Д. Поглощение и рассеяние света малыми частицами. М.: Мир, 1986. 623 с.

[27] Zaitsev A.M. Optical Properties of Diamond. A Data Handbook. Berlin: Springer, 2001. 486 p.

[28] Klein C.A., Hartnett T.M., Robinson C.J.// Phys. Rev. B. 1992. V. 45. N 22. P. 12854-12863. doi 10.1103/PhysRevB.45.12854

[29] Windl W., Pavone P., Karch K., Schutt O., Strauch D., Giannozzi P., Baroni S. // Phys. Rev. B. 1993. V. 48. N 5. P. 3156-3163. doi 10.1103/PhysRevB.48.3156 